RESEARCH AND PRACTICE IN ANESTHESIOLOGY

\section{Case Series}

"Corresponding author

Majed A. Refaai, MD

Associate Professor

Pathology and Laboratory Medicine

University of Rochester Medicine

601 Elmwood Ave. Box-608

Rochester, NY 14642, USA

Tel. 585-276-3927

E-mail: m.refaai@rochester.edu

Volume 1 : Issue 1

Article Ref. \#: 1000AOJ1105

\section{Article History}

Received: March 15 ${ }^{\text {th }}, 2016$

Accepted: April 30 $30^{\text {th }}, 2016$

Published: May $2^{\text {nd }}, 2016$

\section{Citation}

Patel N, Conley GW, McElroy LA, Refaai MA. Isolated prolonged activated partial thromboplastin time and contact factor deficiencies: case series and management review. Res Pract Anesthesiol Open J. 2016; 1(1): 1923. doi: 10.17140/RPAOJ-1-105

\section{Copyright}

(C)2016 Refaai MA. This is an open access article distributed under the Creative Commons Attribution 4.0 International License (CC BY 4.0), which permits unrestricted use, distribution, and reproduction in any medium, provided the original work is properly cited.

\section{Isolated Prolonged Activated Partial Thromboplastin Time and Contact Factor Deficiencies: Case Series and Management Review}

\author{
Nisha Patel, DO'; Grace W. Conley, BS'; Laura A. McElroy, MD²; Majed A. Refaai, MD ${ }^{1 *}$ \\ ${ }^{1}$ Department of Pathology and Laboratory Medicine, University of Rochester Medicine, \\ Rochester, NY, USA \\ ${ }^{2}$ Department of Anesthesiology, University of Rochester Medicine, Rochester, NY, USA
}

\section{ABSTRACT}

Background: Contact factor deficiencies are rare disorders that can cause grossly prolonged activated partial thromboplastin time (aPTT) and activated clotting time (ACT) while rarely affecting in vivo hemostasis. This in vitro laboratory phenomenon poses a particular challenge in surgical procedures that require anticoagulation monitoring.

Case: Here we report two cases of contact factor deficiencies; a 67-year-old morbidly obese female with factor XII deficiency requiring revascularization of a graft and a 58-year-old female with prekallikrein (PK) deficiency undergoing routine muscle biopsy.

Conclusion: Peri-operative anticoagulation monitoring poses a significant challenge in contact factor deficiency patients. Awareness of the challenges of contact factor deficiencies allows for optimal peri-operative management. Emerging literature supports that contact factors play a role in fibrinolysis. Increased surveillance of thrombotic events as well as avoidance of fibrinolytics may be necessary in these patients.

KEYWORDS: Prekallikrein (PK), Factor XII, Activated partial thromboplastin time (aPTT), Contact factors; Fibrinolysis; Anticoagulation; Prolonged clotting time; Thrombosis.

\section{CASE 1}

A 67-year-old morbidly obese $(\mathrm{BMI}=49.3)$ white female, status post recent aortobifemoral bypass, was transferred to our medical center for an emergent right axillary to bifemoral bypass following a newly diagnosed thrombosis of her graft. The patient had no previous records in our health system and limited preoperative laboratory workup revealed an isolated prolonged activated partial thromboplastin time (aPTT) (HemosIL ${ }^{\mathrm{TM}}$ SynthASil on ACL TOP 500 CTS, Instrumentation Laboratory, Bedford, MA, USA) of $>200$ seconds (normal range 25.8-37.9 seconds), which was attributed to the UFH administration during her outside hospitalization. The patient was brought emergently to the operating room where her baseline activated clotting time (ACT) (Hemochron ${ }^{\circledR}$, International Technidyne Corporation; Edison, NJ, USA) was 567 seconds (normal range 105-167 seconds; with a target therapeutic level of 300-400 seconds); suggesting adequate anticoagulation. During surgery, the ACT was repeated twice at 1 and 2 hours and results were consistent with adequate anticoagulation (524 and 480 seconds, respectively) without the use of UFH.

Thirty-seven minutes later, following the 2-hour ACT, a thrombus was detected and the surgeon requested an immediate bolus dose of 10,000 units of UFH. ACT was then repeated and found to be $>1500$ seconds, exceeding the assay detectable limit. Instrument malfunctioning was suspected; however, a repeated ACT test on a different analyzer revealed similar results. Both analyzers were evaluated intra-operatively by a point of care quality assurance 


\section{RESEARCH AND PRACTICE IN ANESTHESIOLOGY}

supervisor and were found to be performing within the standard limits. Intra-operative hemostasis was further managed by empirically dosing the patient with1000 unit/hour of UFH. Surgery was concluded 5 hours later without further complications and with a final postoperative ACT of 586 seconds. The patient's post-operative course was unremarkable with no incidence of excessive bleeding or thrombotic events.

Post-operative workup was initiated to identify the underlying etiology contributing to her abnormal laboratory findings. In addition to her peripheral vascular disease, her past medical history revealed hypertension, chronic obstructive pulmonary disease with an incidental left lower lung lobe mass (later found to be a moderately to well-differentiated squamous cell carcinoma), hyperlipidemia, bipolar disorder, and vitiligo. The patient had no family history of excessive bruising or bleeding and she had previously undergone surgical interventions without bleeding complications. Laboratory workup of the isolated prolonged aPTT is shown in Table 1. UFH effect was ruled out by Hepzyme (Siemens, Marburg, Germany); an enzyme that neutralizes UFH in the plasma sample. Lupus antibody panel (Instrumentation Laboratory) was performed and found to be negative. Mixing studies showed immediate correction of aPTT with a slight prolongation at 1 hour, suspicious for a factor deficiency. Further intrinsic factor analysis (Instrumentation Laboratory) revealed a FXII level of $0.0 \mathrm{U} / \mathrm{mL}$ (normal range $0.57-1.63 \mathrm{U} / \mathrm{mL}$ ) with all other intrinsic factors within normal limits except a high FVIII level. FXII dilutions ruled out a specific factor inhibitor (Table 1). The patient was diagnosed with FXII deficiency and discharged on post-operative day 15 .

\section{CASE 2}

A 53-year-old African American female with a past medical history of hypertension presented to her primary care physician with diffuse myalgias, elevated creatine kinase levels of $976 \mathrm{U} /$ $\mathrm{mL}$ (reference range 34-145 U/mL) (Roche/Hitachi analytes, Indianapolis, IN, USA), and a positive anti-nuclear antibody at 1:360 with nucleolar staining (Zeus Scientific, Branchburg, NJ, USA). Muscle biopsy was recommended and the routine blood workup revealed a normal prothrombin time (PT) and a markedly prolonged aPTT (Instrumentation Laboratory) of $>200$ seconds (Table 2). The patient has no previous history of a prolonged aPTT and no recent history of anticoagulation use. She denied any history of excessive bleeding, bruising, or epistaxis. She reported having an older sister with an "abnormal coagulation disorder", but no other known family history of bleeding or thrombosis.

Lupus antibody panel was performed as part of the prolonged aPTT workup and was found to be negative. The aPTT mixing study showed immediate correction without subsequent prolongation, indicating a factor deficiency. Factor analysis revealed normal levels of FXII, FXI, FIX, and FVIII (Table 2). These findings suggested a deficiency in an upstream precursor of the intrinsic pathway such as HMWK or PK, both which would require a send out to a reference laboratory. During this time, our lab performed a PK screening test utilizing prolonged pre-incubation times with aPTT reagents as previously described. ${ }^{1}$ Briefly, patient plasma sample is pre-incubated with an activator (silica in our case) (STAGO Compact, Diagnostica

\begin{tabular}{|c|c|c|c|c|}
\hline Test & Normal Range & Units & Pre-operative & Post-operative \\
\hline Prothrombin Time (PT) & $9.2-12.3$ & Seconds & 13.6 & 10.4 \\
\hline INR & $1.0-1.2$ & - & 1.3 & 1.0 \\
\hline $\begin{array}{l}\text { Activated Partial Thromboplastin } \\
\text { Time(aPTT) }\end{array}$ & $25.8-37.9$ & Seconds & $>200$ & $>200$ \\
\hline aPTT with Hepzyme & $25.8-37.9$ & Seconds & - & $>200$ \\
\hline Lupus Anticoagulant & Negative & Seconds & - & Negative \\
\hline \multicolumn{5}{|l|}{ aPTT mixing study } \\
\hline - at $0 \mathrm{~min}$ & $25.8-37.9$ & Seconds & - & 36.0 \\
\hline -at $30 \mathrm{~min}$ & $25.8-37.9$ & Seconds & - & 37.3 \\
\hline - at $60 \mathrm{~min}$ & $25.8-37.9$ & Seconds & - & 40.3 \\
\hline Fibrinogen & $172-409$ & $\mathrm{mg} / \mathrm{dL}$ & - & 563 \\
\hline Thrombin Time & $11.7-15.0$ & Seconds & - & 15.6 \\
\hline FVIII & $0.68-1.56$ & $\mathrm{U} / \mathrm{mL}$ & - & 3.12 \\
\hline FIX & $0.92-1.61$ & $\mathrm{U} / \mathrm{mL}$ & - & 1.59 \\
\hline FXI & $0.60-1.54$ & $\mathrm{U} / \mathrm{mL}$ & - & 0.95 \\
\hline FXII & $0.57-1.63$ & $\mathrm{U} / \mathrm{mL}$ & - & 0.0 \\
\hline - at dilution (1:10) & & & - & 0.01 \\
\hline - at dilution (1:20) & & & - & 0.0 \\
\hline - at dilution (1:40) & & & - & 0.0 \\
\hline
\end{tabular}

Table 1: Preoperative and postoperative laboratory findings of case 1 patient. 


\section{RESEARCH AND PRACTICE IN ANESTHESIOLOGY}

\begin{tabular}{|l|c|c|c|}
\hline \multicolumn{1}{|c|}{ Test } & Normal Range & Units & Results \\
\hline PT & $9.2-12.3$ & Seconds & 11.6 \\
\hline aPTT & $25.8-37.9$ & Seconds & $>200$ \\
\hline aPTT with Hepzyme & $25.8-37.9$ & Seconds & $>200$ \\
\hline Lupus Anticoagulant & & Negative & Negative \\
\hline aPTT mixing study & & & \\
\hline - at 0 min & $25.8-37.9$ & Seconds & 36.3 \\
\hline - at 30 min & $25.8-37.9$ & Seconds & 35.6 \\
\hline - at 60 min & $25.8-37.9$ & Seconds & 37.2 \\
\hline FVIII & $0.68-1.56$ & $\mathrm{U} / \mathrm{mL}$ & 0.88 \\
\hline FIX & $0.92-1.61$ & $\mathrm{U} / \mathrm{mL}$ & 1.28 \\
\hline FXI & $0.60-1.54$ & $\mathrm{U} / \mathrm{mL}$ & 1.22 \\
\hline FXII & $0.57-1.63$ & $\mathrm{U} / \mathrm{mL}$ & 0.74 \\
\hline
\end{tabular}

Table 2: Laboratory findings of case 2 patient

Stago, Parsippany, NJ, USA) for a longer period of time prior to the re-calcification step; resulting in a normalization of aPTT. This unique in vitro phenomenon is thought to be due to the auto activation of FXII in the absence of PK. ${ }^{1,2}$ In our case, the patient's plasma was incubated at the aPTT standard incubation time of 240 seconds and then subsequently at 480 seconds, 960 seconds, and 1440 seconds prior to re-calcifications with final aPTT values of $>235,140.1,67.8$, and 53.6 seconds, respectively (Figure 1). Our results yielded the characteristic normalization of aPTT suspicious of PK deficiency. In addition to our qualitative screening studies, PK deficiency was also quantitatively confirmed to be $<15 \%$ (normal range $55-207 \%$ ) by a reference laboratory utilizing STA-R Evolution (Diagnostica Stago, France). The patient underwent a routine muscle biopsy without any complications. Of note, one year later our patient experienced bilateral pulmonary embolisms with a negative malignancy workup, and was placed on warfarin.

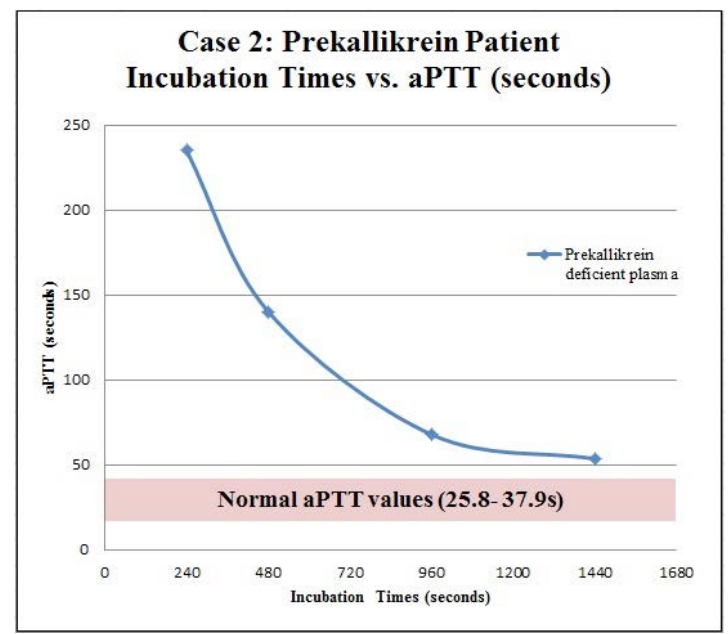

Figure 1: Incubation times versus aPTT (seconds) of second patient (PK deficiency). Patient plasma was preincubated prior to the addition of calcium with silica activator for a series of different times, and aPTT was subsequently measured after addition of calcium. The times measured were $240 \mathrm{sec}-$ onds (standard time), 480 seconds, 960 seconds, and 1440 seconds yielding aPTTs of $>235,140,67.8$, and 53.6 seconds, respectively. Results below show a characteristic normalization curve of aPTT with prolonged incubations suspicious of PK deficiency.

\section{DISCUSSION}

Contact factor deficiencies are rare disorders that are usually detected incidentally during peri-operative screening. As opposed to the other intrinsic coagulation factor deficiencies (FVIII, FIX, and FXI), which cause hemorrhagic diathesis, contact factor deficiencies do not cause excessive bleeding. ${ }^{3}$ Contact factors are however essential for in vitro initiation of coagulation screening tests utilizing negatively charged surfaces, such as aPTT and $\mathrm{ACT}^{3}$ A grossly prolonged aPTT ( $>120$ seconds) is very common in contact factor deficiencies as compared to the downstream intrinsic factor deficiencies (i.e. FXI, FIX, or FVIII). Thus, a contact factor deficiency interference with ACT poses a greater challenge in surgical or invasive medical procedures that require UFH monitoring.

\section{Factor XII Deficiency}

The pathogenesis of FXII deficiency can be either congenital or acquired. The former is usually inherited autosomal recessively. ${ }^{4}$ Acquired FXII deficiency is commonly seen in nephrotic syndrome but has also been described in autoimmune entities, malignancy, and after liver transplantation. ${ }^{5}$ The prevalence of FXII deficiency is unknown, as most patients remain asymptomatic and undiagnosed. Epidemiologic literature studies estimate a $1-3 \%$ prevalence, ${ }^{6}$ with a higher incidence reported in patients with coronary heart disease as high as $10 \%{ }^{7} \mathrm{FXII}$ deficiency is also linked to high incidences of thrombosis (e.g., acute coronary syndrome, miscarriages, and deep venous thrombosis). ${ }^{7,8}$

\section{Prekallikrein Deficiency}

PK deficiency can be either acquired or inherited; the latter is generally autosomal recessive. ${ }^{9} \mathrm{PK}$ is also essential for in vitro initiation of the intrinsic pathway of the coagulation cascade. As discussed in the second case, the grossly prolonged aPTT in PK deficiency can be normalized by a prolonged incubation with the aPTT activator before the re-calcification step. ${ }^{1}$ This phenomenon is believed to be due to the auto-activation of FXII. 


\section{RESEARCH AND PRACTICE IN ANESTHESIOLOGY}

PK plays a significant role in fibrinolysis through the activation of Kallikrein which can convert plasminogen to plas$\min .{ }^{10}$ As in FXII, PK deficiency has been associated with increased risk of arterial and venous thrombosis. Evidence recommends the avoidance of anti fibrinolytics in these patients due to their potential underlying impaired fibrinolysis. ${ }^{11}$

\section{Peri-operative Monitoring of UFH in Contact Factor Deficiency Patients}

Numerous approaches have been suggested to monitor UFH in these patients peri-operatively, ${ }^{11,12}$ including empiric dosing of UFH without monitoring anticoagulation effect, ${ }^{13}$ measuring UFH concentrations directly through blood heparin concentrations utilizing a protamine titration protoco ${ }^{14}$ or indirectly by anti-Xa levels, ${ }^{15}$ administration of fresh frozen plasma (FFP) to obtain a normal baseline ACT and then subsequent monitoring of ACT levels, ${ }^{11,16}$ and more recently using a modified ACT test utilizing in vitro mixing of patient samples with FFP to calculate normal ACT values. ${ }^{17}$ Each method inherently has its own advantages and limitations and the optimal strategy is based on the complexity of the surgical procedure as well as the hemodynamic status of the patient.

The simplest and most commonly used method is empirically dosing the patient with UFH without monitoring (i.e., $\mathrm{ACT}$ ) during the procedure. ${ }^{13,16}$ Once the procedure is completed, UFH anticoagulation effect can then be reversed by protamine sulfate. Although abnormal, a baseline ACT may be helpful in this method to target the UFH reversal.

Utilization of anti-Xa levels was also used to indirectly monitor UFH. However, this method is labor intensive, time consuming and expensive. ${ }^{11}$ Blood heparin levels using a protamine titration curve can be measured in the peri-operative period. This is also a time consuming method and requires special instrumentation such as Hepcon (Medtronic Perfusion Systems, Minneapolis, Minnesota, USA) that may not be readily available. $^{14}$

Peri-operatively transfusion of FFP is another common method used to normalize baseline aPTT and ACT values. Administration of FFP to increase FXII levels was first proposed by Wood et al. and has been successfully implemented in several procedures. ${ }^{11,16}$ It is estimated that an initial dose of $10 \mathrm{~mL} / \mathrm{kg}$ of FFP should raise all coagulation factor levels by about 0.25 $\mathrm{U} / \mathrm{mL}$ unless the patient is bleeding or experiencing an active thrombosis and/or sepsis that surges coagulation factor consumption (e.g., disseminated intravascular coagulopathy - DIC). Thus, an estimated starting dose in a $70-\mathrm{kg}$ patient with FXII or PK levels of $0.0 \mathrm{U} / \mathrm{mL}$ would be approximately 5-6 units of FFP, which is clinically impractical. Furthermore, $50 \%$ of this dose may need to be repeated every two days in some cases where a longer monitoring of UFH is required (FXII and PK half-life is 40-50 and 58 hours, respectively). It is questionable that infusing such large volume of FFP solely for anticoagulation moni- toring without any known therapeutic advantage outweighs the risk of rare but serious transfusion-related complications such as transfusion associated circulatory overload (TACO), transfusion related acute lung injury (TRALI), and infection. This method is best considered for those patients at high risk of thrombosis (e.g., malignancy, previous thrombotic events) and/or low weight patients who require close peri-operative monitoring of UFH.

To avoid exposure to FFP a modified ACT method was proposed by Gerhardt et al. ${ }^{17}$ In this method, an individualized dose of FFP is added to an ex vivo blood sample prior to establishing a baseline ACT. This method however is time consuming, requiring preoperative generation of a patient-specific FFP curve, and the ACT values may not be accurate due to differences in ATIII levels in both FFP and patient plasma. ${ }^{17}$

In addition, viscoelastic tests, such as thromboelastography (TEG), have also been studied in cases of contact factor deficiencies, however they yield mixed results. In one study of PK deficiency, TEG parameter values failed to detect a contact factor deficiency whereas ACT and aPTT were abnormal. ${ }^{14}$

In summary, we report two case reports of contact factor deficiencies incidentally diagnosed during peri-operative workup. In case 1, empiric dosing of heparin intra operatively allowed for successful continuation of bypass graft surgery. In case 2 , routine muscle biopsy was performed successfully without any significant adverse events. Coincidentally, both patients experienced thrombotic events during the time of having a deficient coagulation factor, supporting the notion that these patients are not protected against thrombotic events at may be at risk for prothrombotic tendencies. Thus contact factor deficiencies are a rare but important differential diagnosis for an isolated prolonged aPTT that must be recognized early for anticoagulation monitoring and surveillance of thrombotic events.

\section{AUTHORSHIP CONTRIBUTIONS}

N. Patel and M.A. Refaai analyzed the data and drafted the manuscript. G.W. Conley and L.A. McElroy contributed to the collection and interpretation of the data and critical review of the manuscript.

\section{CONFLICTS OF INTEREST}

The authors do not declare any conflicts of interests.

\section{REFERENCES}

1. Asmis LM, Sulzer I, Furlan M, Lammle B. Prekallikrein deficiency: the characteristic normalization of the severely prolonged aPTT following increased preincubation time is due to autoactivation of factor XII. Thromb Res. 2002; 105(6): 463470. doi: 10.1016/S0049-3848(02)00045-2 


\section{RESEARCH AND PRACTICE IN ANESTHESIOLOGY}

2. Schmaier AH. The elusive physiologic role of factor XII. $J$ Clin Invest. 2008; 118(9): 3006-3009. doi: 10.1172/JCI36617

3. Renne T, Schmaier AH, Nickel KF, Blomback M, Maas C. In vivo roles of factor XII. Blood. 2012; 120(22): 4296-4303. doi: 10.1182/blood-2012-07-292094

4. Sonnenfeld H, Rousseau J, Leroy B, Scherpereel P. Congenital factor XII deficiency: a rare cause of increased activated cephalin time. Ann Fr Anesth Reanim. 1985; 4(4): 378-379. doi: 10.1016/S0750-7658(85)80110-6

5. Shin DY, Lee HR, Kang HJ, Na, II, Chang YH, Yang SH. Prevalent factor XII deficiency in cancer patients with isolated aPTT prolongation. Blood Research. 2015; 50(2): 114-117. doi: $10.5045 /$ br.2015.50.2.114

6. Halbmayer WM, Haushofer A, Schon R, Mannhalter C, Strohmer E, Baumgarten K, Fischer M. The prevalence of moderate and severe FXII (Hageman factor) deficiency among the normal population: evaluation of the incidence of FXII deficiency among 300 healthy blood donors. Thromb Haemost. 1994; 71(1): 68-72. Web site. http://europepmc.org/abstract/ med/8165648. Accessed.

7. Halbmayer WM, Haushofer A, Radek J, Schon R, Deutsch M, Fischer M. Prevalence of factor XII (Hageman factor) deficiency among 426 patients with coronary heart disease awaiting cardiac surgery. Coronary Artery Disease. 1994; 5(5): 451-454. Web site. http://journals.lww.com/coronary-artery/Abstract/1994/05000/Prevalence_of_factor_XII_Hageman_factor_.12.aspx. Accessed.

8. Halbmayer WM, Mannhalter C, Feichtinger C, Rubi K, Fischer M. The prevalence of factor XII deficiency in 103 orally anticoagulated outpatients suffering from recurrent venous and/or arterial thromboembolism. Thromb Haemost. 1992; 68(3): 285290. Web site. http://europepmc.org/abstract/med/1440493. Accessed.

9. Sollo DG, Saleem A. Prekallikrein (Fletcher factor) deficiency. Ann Clin Lab Sci. 1985; 15(4): 279-285. Web site. http:// www.annclinlabsci.org/content/15/4/279.short. Accessed

10. Colman RW. Activation of Plasminogen by Human Plasma Kallikrein. Biochem Bioph Res Co. 1969; 35(2): 273-279. doi: 10.1016/0006-291X(69)90278-2

11. Conaglen PJ, Akowuah E, Theodore S, Atkinson V. Implications for cardiac surgery in patients with factor XII deficiency. Ann Thorac Surg. 2010; 89(2): 625-626. doi: 10.1016/j.athoracsur.2009.07.042

12. DeBois W, Liu J, Lee L, et al. Cardiopulmonary bypass in patients with pre-existing coagulopathy. J Extra Corpor Technol. 2005; 37(1): 15-22. Web site. http://www.ncbi.nlm.nih.gov/
pmc/articles/PMC4680798/. Accessed.

13. Kelsey PR, Bottomley J, Grotte GJ, Maciver JE. Congenital factor XII deficiency: successful open heart surgery and anticoagulation. Clin Lab Haematol. 1985; 7(4): 379-381. doi: 10.1111/j.1365-2257.1985.tb00053.x

14. Cankovic L, Steenwyk BL, McGiffin DC, Nielsen VG. Practical approach to anticoagulation for cardiopulmonary bypass in the patient with congenital prolonged activated partial thromboplastin time. Blood Coagul Fibrinolysis. 2008; 19(7): 725-726. doi: 10.1097/MBC.0b013e32830891ab

15. van Veen JJ, Laidlaw S, Swanevelder J, et al. Contact factor deficiencies and cardiopulmonary bypass surgery: detection of the defect and monitoring of heparin. Eur J Haematol. 2009; 82(3): 208-212. doi: 10.1111/j.1600-0609.2008.01191.x

16. Wood MK. Congenital factor XII deficiency and cardiopulmonary bypass. Ann Thorac Surg. 1994; 58(5): 1565. doi: 10.1016/0003-4975(94)91976-3

17. Gerhardt MA, Greenberg CS, Slaughter TF, Stafford Smith M. Factor XII deficiency and cardiopulmonary bypass: use of a novel modification of the activated clotting time to monitor anticoagulation. Anesthesiology. 1997; 87(4): 990-992. Web site. http://anesthesiology.pubs.asahq.org/article.aspx?articleid= 1948727. Accessed. 